\title{
Impacto da saúde bucal na qualidade de vida do idoso
}

\author{
The impact of Oral Health related quality of life in \\ elderly
}

Impacto de la salud bucal en la calidad de la vida de los ancianos

Simone Kreve

Didier Anzolin

RESUMO: A preocupação com a qualidade de vida na terceira idade é relevante. Perdas dentárias podem ter efeitos significativos na saúde e na vida dos indivíduos, pois afetam a capacidade mastigatória, restringem o consumo de diversos alimentos, dificultam a fonação, além de causar danos estéticos. O objetivo desta revisão da literatura é discutir as alterações na qualidade de vida do paciente idoso, incluindo os efeitos psicológicos, a percepção dos idosos sobre saúde bucal, e o reflexo emocional.

Palavras-chave: Reabilitação bucal; Satisfação do paciente; Qualidade de vida.

ABSTRACT: Concern about quality of life in the elderly individuals is relevant. Dental loss cause significant effects on subjects health and life because it affects the chewing capacity, restricts some food consumption, phonation difficult and cause aesthetic damage. The aim of this literature review is discuss the changes in elderly patient quality of life, Including psychological effects, the perception of the elderly on oral health and the emotional reflex.

Keywords: Mouth Rehabilitation; Patient Satisfaction; Quality of life. 
RESUMEN: Una preocupación por la calidad de la vida en la tercera edad es relevante. Perdas dentarias pueden tener efectos significativos en la salud y en la vida de los individuos, para la determinación de la capacidad, la restricción o el consumo de diversos alimentos, la dificultad de fonación y el daño causado por los factores estéticos. El objetivo de la revisión de la literatura es discutir como los cambios en la calidad de la vida del paciente, incluyendo los efectos psicológicos, la percepción de los ancianos sobre salud bucal y el reflejo emocional.

Palabras-clave: Rehabilitación Bucal; Satisfacción del Paciente; Calidad de Vida.

\section{Introdução}

A Odontologia atual parte de um contexto de formação profissional que procura atender os pacientes sem focar somente a queixa odontológica. Assim, as particularidades de cada indivíduo que podem interferir nas reações sistêmicas do organismo passam a ser levadas em consideração no momento da realização de um diagnóstico e plano de tratamento.

A atenção ao paciente idoso que, por fazer parte de um grupo em cujo histórico médico se encontram algumas doenças ou limitações orgânicas frequentemente associadas ao envelhecimento, deve receber assistência de uma equipe multidisciplinar (Domingos, Moratelli, \& Oliveira, 2011). Levando em conta que a saúde bucal é um componente de importância global, deve-se ressaltar sua inclusão nos quesitos de avaliação de saúde geral considerando esta um potencial na qualidade de vida como um todo (Yiengprugsawan, Somkotra, Seubsman, \& Sleigh, 2011).

A perda de um dente está associada com impactos estéticos, funcionais, psicológicos e sociais, além de ser considerado um sério problema de saúde pública, já que atinge diretamente a autoestima (Okoje, Dosumu, Alonge, \& Onyeaso, 2012; Goursand, Rocha, \& Almeida, 2014; Agostinho, Campos, \& Silveira, 2015). Considerando a ausência ou rara existência de programas odontológicos voltados para idosos, a Odontologia necessita de uma prática de atendimento para esse grupo populacional, seja preventiva seja curativa, com uma visão acurada sobre o que é ser idoso, e sobre o envelhecer em geral. 
Apesar de ser um processo natural, o envelhecimento é caracterizado por alterações fisiológicas (alterações da pele, ossos e perda de massa muscular, gerando diminuição de peso e dificuldade na marcha), bioquímicas e psicológicas, que levam geralmente a uma diminuição da capacidade de adaptação do indivíduo ao meio ambiente (Goursand, Rocha, \& Almeida, 2014).

Os idosos têm sido foco de atenção principalmente quanto ao impacto da saúde bucal em sua qualidade de vida. Pensando justamente no bem-estar físico, mas especialmente mental, fica a dúvida quanto à importância das reabilitações protéticas para pacientes idosos.

Será que as intervenções tal como costumam acontecer funcionam, na verdade? Será que um tratamento protético que tem sucesso funcional em indivíduos edêntulos (com perda total de dentes), tem similar "sucesso psicológico"?

Embora a terapia com prótese total convencional não seja considerada o padrão de tratamento para o edentulismo, em alguns países desenvolvidos, este ainda é amplamente utilizado como repositor da dentição ausente, e não se espera que o uso do mesmo venha a declinar em futuro próximo, especialmente em populações menos desenvolvidas, com recursos econômicos limitados (Hantash, Al-Omiri, Yunis, DarOdeh, \& Lynch, 2011).

A reabilitação do paciente edêntulo possibilita aspectos que contribuem significativamente na sua nutrição, fonética, relações sociais, autoestima e consequentemente na melhora da saúde geral e sua qualidade de vida. Entre outras posturas, o paciente geriátrico não mais aceita o tratamento odontológico baseado em exodontias e próteses totais removíveis e passa a ter aspirações estéticas de que antes não dispunha.

Levando em consideração a importância das próteses na mecânica mastigatória (Medeiros, Pontes, \& Magalhães, 2014; Soares, Rodrigues, Ribeiro, \& Rosendo, 2015) e na estética, e consequentemente a influência da reabilitação oral sobre o fator emocional dos pacientes edêntulos, este estudo tem o objetivo de discutir alterações tributárias a vários aspectos: sobrelevam os traumas psicológicos do edentulismo; evidenciam o impacto da reabilitação oral na percepção da autoimagem e, consequentemente, seus resultados na qualidade de vida de uma pessoa idosa. 


\section{Materiais e Métodos}

Foi realizado um levantamento de estudos publicados nas bases de dados SciELO, PubMed, EBSCO, BIREME (BBO e LILACS) e Google Scholar sobre o perfil psicológico do idoso edêntulo, a percepção de qualidade de vida, o impacto da reabilitação na vida do idoso e a autoimagem bucal.

Realizou-se um levantamento bibliográfico por meio de estratégia de busca com base nos seguintes termos de pesquisa: "Reabilitação oral no idoso", "Perfil psicológico", "Perda dentária", "Mastigação", "Percepção" e "Saúde bucal”.

Cada uma dessas palavras-chave foi mencionada de formas diferentes para que pudessem ser recuperados vários estudos. Foram incluídos trabalhos publicados até 2015

Títulos e resumos foram selecionados para que se pudessem obter informações sobre o estudo, sobre a revisão da literatura, tamanho da amostra, e a análise completa do texto foi realizada nas publicações relevantes.

Adotaram-se como critérios de inclusão: artigos publicados em português e inglês, textos completos e anexados nas bases de dados, revisões e pesquisas clínicas.

Utilizaram-se como critérios de exclusão: teses e dissertações, estudos envolvendo grupos de pacientes com doenças específicas, inclusive trabalhos mais antigos que 2011, e estudos com grupos de pacientes portadores de doenças mentais.

Considerando-se as bases científicas analisadas, 21 referências relacionadas ao tema atenderam aos critérios e foram selecionadas.

\section{Revisão de literatura e discussão}

\section{Auto-imagem}

A auto-estima está relacionada com a imagem corporal, principalmente por padrões estéticos impostos pela sociedade. 
É claramente evidenciado na literatura que as perdas dentárias causam alterações físicas na face. Por exemplo, pode-se observar depressão da comissura labial e da base do nariz, perda do tônus muscular, redução da altura vertical do terço inferior da face e aprofundamento das linhas de expressão, além do aparecimento de uma vasta gama de reações psicológicas, que incluem sensação de luto, perda de autoconfiança, preocupação com a aparência e autoimagem.

Todos esses problemas fazem com que o indivíduo mantenha em segredo a perda do dente, vendo-a como um tabu que não deve ser discutido com as demais pessoas (Okoje, et al., 2012).

Quando um paciente procura tratamento para substituição de elementos dentais perdidos, está também procurando a reconstituição de sua imagem pessoal e social (Abreu, \& Munhoz, 2011).

O grau de satisfação de idosos em relação a sua própria saúde bucal foi estudado demonstrando-se que o simples acesso à prótese dentária não foi suficiente para satisfação da condição de saúde bucal (Agostinho, Campos, \& Silveira, 2015).

A reabilitação do edêntulo deve visar a não somente a reposição dos dentes perdidos, mas também proporcionar além de conforto e função mastigatória, a estética almejada por ele.

Em um estudo realizado com pacientes portadores de próteses totais, os autores evidenciaram que os pacientes que mais buscam por reabilitação oral são as mulheres, como também elas demonstram uma maior preocupação com a estética e com o convívio social (Soares, Rodrigues, Ribeiro, \& Rosendo, 2015).

\section{Transtornos psicológicos}

A falta de estética ocasionada pelas perdas dentárias muitas vezes supera a necessidade de função mastigatória. Tal falta de estética pode levar a uma descaracterização facial percebida pelo indivíduo, fazendo com que o mesmo se sinta diminuído socialmente e com quadros de dificuldade de aceitação social plena, tornando-se estigmatizado (Goursand, Rocha, \& Almeida, 2014). 
Estudos realizados por meio de questionário com a população idosa demonstraram que ela percebeu um maior impacto da perda dentária no quesito desconforto psicológico e esteve associada a uma maior chance de comprometimento na qualidade de vida em seus domínios físico e mental (Goursand, Rocha, \& Almeida, 2014; Martins, Jones, Souza, \& Pordeus, 2014). Ou seja, os idosos relataram dificuldade de relaxar, de apreciar as mais diversas situações que se lhes apresentaram devido à falta de dentes.

Alguns autores enfatizam a necessidade de privacidade para o tratamento protético e uma necessidade de preparar os pacientes para o efeito emocional que vinha acompanhando a perda do dente (Okoje, et al., 2012).

Pensando-se, pois, em pacientes edêntulos, a reabilitação oral pode ser o fatorchave para seu sucesso físico e emocional. A necessidade de próteses totais para restabelecer a estética e função pode contribuir para a integração social e melhoria da autoimagem dos pacientes (Papadaki, \& Anastassiadou, 2012; Martins, et al., 2014). Isso pode ser explicado, como dito antes, pelo fato de o desconforto psicológico e a incapacidade psicológica refletirem a falta de estética ocasionada pela perda dentária, superando muitas vezes a função mastigatória.

Justamente pelo fato de o edentulismo implicar sentimentos de aprovação ou rejeição, acaba ele por interferir nos relacionamentos interpessoais (Martins, et al., 2014). Da mesma forma com são notados, no meio social, pacientes indiferentes a seu estado edêntulo, também são percebidos aqueles pacientes odonto-mutilados que se esquivam do meio social, seja pelo fato de se apresentarem sem dentes, seja por estarem insatisfatoriamente reabilitados.

Fatores psicológicos são mais fortemente relacionados com a aceitação e a adaptação da prótese do que com as condições anatômicas e a qualidade da dentadura (Sowmya,Vinaya, \& Krishna, 2011).

Dentre os fatores psicológicos, a depressão, a ansiedade e o medo podem ter efeitos significativos sobre a adaptabilidade ao tratamento protético (Sowmya, Vinaya, \& Krishna, 2011). 
O dentista deve estar a par sobre como lidar com problemas psicológicos, bem como com problemas dentários de seus pacientes. Isso não deve ser interpretado de maneira a substituir o dentista por um psiquiatra, mas o profissional deve aconselhar o paciente e membros de sua família e, quando necessário, encaminhá-lo para uma consulta médica/psicológica (Polsani, Ajay Kumar, Githanjali, \& Anjana, 2011).

Os pacientes têm, via de regra, algum grau de ansiedade no consultório de um dentista, e isso requer que este saiba lidar com os transtornos de ansiedade e comportamentos decorrentes de um tratamento.

A ansiedade tem sido considerada um problema para a manutenção ou obtenção de saúde bucal e, na tentativa de impedir que o indivíduo desenvolva medo do tratamento odontológico, é necessário disponibilizar experiências odontológicas agradáveis.

O medo pode ser definido como uma reação a um perigo conhecido. $\mathrm{O}$ medo do tratamento odontológico é chamado na atualidade de "odontofobia", e tem sua genealogia nas ciências psicossociais e comportamentais. Pacientes apresentam-se frequentemente nervosos, apreensivos, desconfiados, e até mesmo apavorados (Sowmya,Vinaya, \& Krishna, 2011; Bulgarelli, Mestriner, \& Pinto, 2012).

É provável que os pacientes, ao se aproximarem de uma perda dentária grande ou total, apresentem ansiedade e depressão; por isso, a preparação emocional do paciente é tão importante quanto a preparação dos tecidos gengivais.

\section{Interações sociais}

Parâmetros físicos, psicológicos e sociais influenciam os sentimentos em torno de uma doença ou uma incapacidade, bem como a respeito das possibilidades de tratamento (de Souza, et al., 2010). Ocorre na maior parte das vezes um impacto nas atividades diárias de uma pessoa devido a problemas relacionados com a boca, dentes ou próteses (Bittencourt, Abegg, \& Fontanive, 2013).

Observa-se, em trabalhos de alguns autores, que as pessoas mais jovens com problemas dentários sentem dificuldades quanto a interações sociais, enquanto as mais velhas apresentam uma maior dificuldade mastigatória, e também na fala e na deglutição (Yiengprugsawan, et al., 2011). 
É comum observar a colocação de uma das mãos na tentativa de mascarar os dentes ou a ausência deles, seja no meio social, em lar de idosos, encontros da terceira idade, na rua, no ônibus. Muitos pacientes não conseguem se relacionar com outras pessoas pelo fato de sentirem vergonha de pronunciar incorretamente os fonemas.

Os pacientes têm tanto sua vida funcional (física), quanto sua vida social alterada pela não articulação correta das palavras (fator psicossocial) (Abreu, \& Munhoz, 2011).

Vários fatores psicossociais influenciam a percepção subjetiva da qualidade de vida relacionada à saúde oral e devem ser levados em conta no planejamento e posterior avaliação do sucesso do tratamento (Pistorius, J., Horn, Pistorius, A., \& Kraft, 2013).

As preocupações financeiras, o bloqueio ao acesso, questões sociais, culturais e de estilo de vida, são os principais grupos de fatores psicossociais que foram identificados como potenciais barreiras entre necessidade e a procura por atendimento (Sowmya, Vinaya, \& Krishna, 2011; Bulgarelli, Mestriner, \& Pinto, 2012, Agostinho, Campos, \& Silveira, 2015).

Os motivos pelos quais muitos idosos não consultam regularmente o cirurgiãodentista foram assunto de um estudo que demonstrou que, mesmo cadastrados na área de abrangência de uma unidade de saúde da família, os idosos afirmaram precisar de dinheiro para cuidar da saúde bucal, o que demonstra desconhecimento sobre a saúde como direito de todo o cidadão e dever do Estado (Bulgarelli, Mestriner, \& Pinto, 2012).

A perda dentária pode caracterizar um problema importante na comunicação verbal, consequentemente acarreta alteração na vida afetiva e na própria sexualidade, diminuindo, assim, a capacidade funcional dos idosos perante seu grupo social (Goursand, Rocha, \& Almeida, 2014).

\section{Qualidade de vida}

Ao longo das últimas décadas tem se tornado frequente o interesse da saúde bucal relacionada à qualidade de vida (de Souza, et al., 2010). 
Segundo o conceito de Ruffino Netto (1992), uma boa qualidade de vida é aquela que oferece um mínimo de condições para que os indivíduos nela inseridos possam desenvolver o máximo de suas potencialidades, sendo elas: viver, sentir ou amar, trabalhar produzindo bens ou serviços, fazer ciência ou artes, viver para adquirir meios utilitários, ou simplesmente existir.

Assim, a saúde e a qualidade de vida relacionada à saúde podem ser vistas como constituindo um conceito multidimensional representado pela combinação da saúde geral aliada às perspectivas de um paciente (de Souza, et al., 2010).

A abordagem interdisciplinar da atenção ao idoso, no que se refere à saúde bucal, tem se mostrado eficiente ao influenciar na qualidade de vida deste indivíduo por permitir que ele seja tratado dentro de uma visão integrada, sem desconsiderar a ocorrência de doenças sistêmicas que interfiram no ambiente bucal (Domingos, Moratelli, \& Olveira, 2011).

Ao serem analisados qualitativamente questionários acerca de qualidade de vida, em um estudo realizado com idosos institucionalizados, estudiosos observaram que as alterações de deglutição causaram impacto na qualidade de vida desses idosos, que, em sua maior parte, acabaram por não apresentar queixa, e tentaram de maneira espontânea se adaptar às suas limitações por meio da criação de estratégias ou até mesmo da eliminação de certas consistências alimentares (Cardoso, Teixeira, Baltezan, \& Olchik, 2014).

O questionamento aos indivíduos sobre a perda dos dentes, com a finalidade de aumentar o conhecimento e de diagnosticar sua percepção sobre a saúde bucal, foi objeto de estudo de um trabalho realizado na Nigéria. O estudo demonstrou que as indicações para extrações dentárias mais frequentes foram cárie dentária, acidentes de trânsito e quedas, tanto em crianças quanto em adultos. Contudo, as doenças periodontais foram destacadas como as causas mais comuns nas pessoas idosas (Okoje, et al., 2012).

Outro estudo demonstrou que pacientes tabagistas, que fazem uso regular de álcool, e com menos de 20 dentes na boca, apresentaram baixos escores nas pesquisas com indicadores orais que relatam qualidade de vida, ou seja, aumentam-se, nessa condição, as chances de dificuldades na fala, deglutição, mastigação, entre outros (Yiengprugsawan, et al., 2011). 
A inter-relação da saúde bucal com a qualidade de vida pode ser facilmente observada: após uma reabilitação oral adequada os idosos, estes se tornam mais comunicativos, mais participativos, mais proativos, com maior autoestima, capazes de refletir sobre a sua qualidade de vida, e de realizar o autocuidado em saúde (Lima Moreira, Moreira de Moura, de Oliveira Vasconcelos, Lima Verde, \& Brígido de Moura, 2014).

\section{Reabilitação}

A preservação e a recuperação da função mastigatória (Medeiros, Pontes, \& Magalhães Jr., 2014) representam um dos principais objetivos de um tratamento odontológico reabilitador, e vários aspectos são levados em consideração no momento da realização da reabilitação com próteses (Abreu, \& Munhoz, 2011).

Segundo uma pesquisa realizada com portadores de próteses totais, dentre os itens avaliados (dimensão estética e social, eficiência mastigatória e desconforto com o uso da prótese e/ou dor), aquele que trata da eficiência mastigatória apresentou os valores mais elevados, característicos de uma percepção ruim, deixando claro que a maior parte dos entrevistados apontou a dificuldade na mastigação como fator mais importante (Soares, et al., 2015).

Em um estudo realizado por meio de questionários, alguns autores verificaram que quase $60 \%$ dos indivíduos responderam "Sempre" quando foram questionados se nos últimos 12 meses deixaram de comer o que queriam (Francisco, Lacerda, \& Casotti, 2015). Esse fator está intimamente relacionado com questões sociais, já que a capacidade mastigatória alterada impossibilita ou modifica as atividades de alimentação em grupo.

Em uma pesquisa com idosos institucionalizados, referente à situação dentária, metade da população estudada (50\%) apresentava edentulismo (Oliveira, Delgado, \& Brescovici, 2014). Dificuldades na adaptação de próteses e a higiene bucal precária foram os dados coletados, que evidenciaram a falta de programas de promoção e prevenção voltados para esta população. 
Estudos com o objetivo de verificar a auto-percepção de saúde bucal dos idosos demonstraram, de uma forma geral, nos grupos estudados, que a mesma pode ser considerada "ruim", considerando a alta frequência de edentulismo total ou parcial, assim como a necessidade de tratamento com próteses, incluindo as que estavam em uso, mas com perda de função e de estética (Agostinho, Campos, \& Silveira, 2015; Francisco, Lacerda, \& Casotti, 2015).

Detectou-se, em outro estudo, que o uso de prótese total se revela como um motivo pela não-procura por um cirurgião-dentista (Bulgarelli, Mestriner, \& Pinto, 2012). Isso pode ser denotado principalmente pela falta de políticas que incentivem a busca pelo atendimento odontológico, pois pesquisas demonstram que os portadores de próteses totais revelam a crença de que visitar regularmente o dentista é necessário apenas para as pessoas que possuem dentes.

Alguns problemas como dentadura mal ajustada e perda dentária podem estar relacionados à falta de atenção à saúde bucal a que os indivíduos foram submetidos ao longo de toda a vida, possivelmente devido à ausência de serviços odontológicos ou dificuldade de acesso aos mesmos (Bittencourt, Abegg, \& Fontanive, 2013).

Dentes saudáveis se tornaram altamente valorizados na sociedade, e o confronto diário com a colocação e remoção das próteses dentárias, com todas as suas possíveis desvantagens estéticas e funcionais, resultam em grande redução da percepção subjetiva da qualidade de vida (Pistorius, et al., 2013).

A prótese permite a esperança de recuperação de uma aparência desejável. Um belo sorriso pode demonstrar simpatia, alegria e autoconfiança; é capaz de despertar sentimentos, e chamar a atenção. Principalmente despertar o desejo e a vontade de posse de quem não o tem. Em vista disso, a crescente busca de pacientes edêntulos, não apenas por uma reabilitação que devolva função, mas também que lhes devolva um belo sorriso.

A substituição do estado edêntulo para o dentado deve ser acompanhada de técnicas e critérios que visam à satisfação desse paciente (Abreu, \& Munhoz, 2011). Seja esta a satisfação de ter dentes que possibilitam uma ótima função mastigatória, ou a de ter dentes belos que formem um conjunto agradável, que proporcione aspecto jovial, ou ainda a combinação desses fatores (Medeiros, Pontes, \& Magalhães Jr., 2014). 
Existe a premente necessidade de o profissional da odontologia instruir seu paciente, auxiliando-o no enfrentamento de necessários procedimentos dentários, também ser claro quanto às expectativas e o real resultado a ser obtido. O sucesso no tratamento odontogeriátrico nada mais é senão o resultado da construção de confiança entre o paciente e o dentista, independentemente da qualidade final da prótese (Polsani, et al., 2011).

A prótese total convencional pode melhorar a satisfação e a qualidade de vida dos pacientes. Esta ainda é válida, independentemente da técnica utilizada para a sua fabricação (Hantash, et al., 2011).

De uma forma geral, a estética pode ser analisada por dois pontos de vista: o da sociedade, pois cada uma tem seus próprios conceitos de beleza e estética; e do ponto de vista do próprio indivíduo, que também tem suas visões sobre o que lhe é considerado belo e estético.

O rosto é a parte mais diferenciada do corpo e está indissociavelmente ligado ao indivíduo e a sua identidade. $\mathrm{O}$ sorriso tem conotações muito significativas, entre outras, as de bem-estar, alegria, segurança, autossatisfação, satisfação em relação ao outro, mais frequentemente ligado à juventude, beleza, produtividade e possibilidade de realização na vida. Os dentes são considerados aspectos importantes nas relações empregatícias, sociais, culturais, além de influenciar na autoestima das pessoas.

A beleza física é algo almejado dentro da sociedade, e falando particularmente da Odontologia, mais precisamente no tratamento com prótese total convencional, a beleza dos dentes, o conjunto formado entre uma prótese total bem confeccionada e o arranjo muscular, faz com que a procura, que antes era estritamente funcional, se torne potencialmente estética. E para que esse desejo seja cumprido, o dentista deve estar ciente da preocupação de que o paciente tem em relação a sua aparência, e se esforçar para atender às exigências estéticas (Polsani, et al., 2011).

\section{Conclusão}

Podemos concluir com esta revisão que é necessário trabalhar a educação em saúde para que os idosos sejam conscientizados da necessidade de encontrar tempo, de superar o medo e de reconhecer a importância de cuidar da saúde bucal. 
Dessa forma, prevenir a perda dentária, reconhecer e tratar apropriadamente as necessidades no que diz respeito à capacidade mastigatória e aparência bucal, dado que estas têm forte impacto no bem-estar geral dos idosos. É necessário que o paciente entenda que o sucesso da reabilitação protética é um processo que requer tempo e paciência, sendo imprescindível visitar regulamente o dentista e fazer o uso correto de uma prótese.

\section{Referências}

Abreu, C. W. de, \& Munhoz, E. (2011). Os fatores que influenciam na satisfação do paciente submetido a tratamento de prótese total convencional. HU Rev, 37(4), 413-419. Recuperado em 01 junho, 2015, de: file:///C:/Users/Dados/Downloads/1559-9571-1-PB.pdf.

Agostinho, A. C. M. G., Campos, M. L., \& Silveira, J. L. G. C. D. (2015). Edentulismo, uso de prótese e autopercepção de saúde bucal entre idosos. Rev Odontol UNESP, 44(2), 74-79. Recuperado em 01 dezembro, 2015, de: doi: http://dx.doi.org/10.1590/1807-2577.1072.

Bittencourt, V., Abegg, C., \& Fontanive, V. N. (2013). O impacto da saúde bucal nas atividades diárias de indivíduos de 50 a 74 anos em três distritos sanitários de Porto Alegre/RS. Revista da Faculdade de Odontologia-UPF, 18(1), 37-43. Recuperado em 01 junho, 2015, de: file:///C:/Users/Dados/Downloads/2858-12651-2-PB.pdf.

Bulgarelli, A. F., Mestriner, S. F., \& Pinto, I. C. (2012). Percepções de um grupo de idosos frente ao fato de não consultarem regularmente o cirurgião-dentista. Revista Brasileira de Geriatria e Gerontologia, 15(1), 97-107. Recuperado em 01 junho, 2015, de: http://dx.doi.org/10.1590/S1809-98232012000100011.

Cardoso, S. V., Teixeira, A. R., Baltezan, R. L., \& Olchik, M. R. (2014). O impacto das alterações de deglutição na qualidade de vida de idosos institucionalizados. São Paulo, SP: Kairós Gerontologia, 17(1), 231-245. Recuperado em 01 junho, 2015, de: file:///C:/Users/Dados/Downloads/21196-54155-1-SM.pdf.

de Souza, R. F., Terada, A. S. S. D., Vecchia, M. P. D., Regis, R. R., Zanini, A. P., \& Compagnoni, M. A. (2010). Validation of the Brazilian versions of two inventories for measuring oral health-related quality of life of edentulous subjects. Gerodontology, 29(2), e88-e95. Recuperado em 01 junho, 2015, de: doi: 10.1111/j.17412358.2010.00417.x.

Domingos, P. A. D. S., Moratelli, R. D. C., \& Oliveira, A. L. B. M. D. (2011). Atenção odontológica integral ao idoso: uma abordagem holística. Rev. Odontol. Univ. Cid. São Paulo (on-line), 23(2), 143-153. Recuperado em 01 junho, 2015, de: http://files.bvs.br/upload/S/1983-5183/2011/v23n2/a2262.pdf.

Francisco, K. M. S., Lacerda, T. C., \& Casotti, C. A. (2015). Autopercepção de saúde bucal de idosos. Clínica e Pesquisa em Odontologia-UNITAU, 7(1), 2-8. Recuperado em 01 dezembro, 2015, de: http://periodicos.unitau.br/ojs2.2/index.php/clipeodonto/article/viewFile/2011/1526. 
Goursand, D., Rocha, E. A., \& Almeida, P. S. (2014). O impacto gerado pelas ausências dentárias nos idosos. Clínica e Pesquisa em Odontologia-UNITAU, 6(1), 46-53. Recuperado em 01 junho, 2015, de: http://periodicos.unitau.br/ojs2.2/index.php/clipeodonto/article/viewFile/1876/1396.

Hantash, R. O. A., AL-Omiri, M. K., Yunis, M. A., Dar-Odeh, N., \& Lynch, E. (2011). Relationship between impacts of complete denture treatment on daily living, satisfaction and personality profiles. The Journal of Contemporary Dental Practice, 12(3), 200-207. Recuperado em 01 junho, 2015, de:

http://www.jaypeejournals.com/eJournals/ShowText.aspx?ID=2224\&Type=FREE\&TY $\mathrm{P}=\mathrm{TOP} \& \mathrm{IN}=\_$eJournals/images/JPLOGO.gif \&IID=186\&isPDF=YES.

Lima Moreira, A. C., Moreira de Moura, P. M. M., de Oliveira Vasconcelos, M., Lima Verde, M. E. Q., \& Brígido de Moura, W. V. (2015). Os 15 anos do projeto sorriso grisalho e os avanços no campo da odontogeriatria. Extensão em Ação, 2(7), 98-110. Recuperado em 01 dezembro, 2015, de:

http://www.revistaprex.ufc.br/index.php/EXTA/article/view/174.

Martins, A. M. E. B. L., Jones, K. M., Souza, J. G. S., \& Pordeus, I. A. (2014). Associação entre impactos funcionais e psicossociais das desordens bucais e qualidade de vida entre idosos. Ciência \& Saúde Coletiva, 19(8), 3461-3478. Recuperado em 01 junho, 2015, de: http://dx.doi.org/10.1590/1413-81232014198.16202013.

Medeiros, S. L. D., Pontes, M. P. D. B., \& Magalhães Jr., H. V. (2014). Autopercepção da capacidade mastigatória em indivíduos idosos. Revista Brasileira de Geriatria e Gerontologia, 17(4), 807-817. Recuperado em 01 junho, 2015, de: http://dx.doi.org/10.1590/1809-9823.2014.13150.

Okoje, V. N., Dosumu, O. O., Alonge, T. O., \& Onyeaso, C. (2012). Tooth loss: are the patients prepared? Nigerian Journal of Clinical Practice, 15(2), 172-175. Recuperado em 01 junho, 2015, de: doi: 10.4103/1119-3077.97305.

Oliveira, B. S. D., Delgado, S. E., \& Brescovici, S. M. (2014). Alterações das funções de mastigação e deglutição no processo de alimentação de idosos institucionalizados. Rev Bras Geriatr Gerontol, 17(3), 575-587. Recuperado em 01 junho, 2015, de: http://www.redalyc.org/pdf/4038/403838839011.pdf.

Papadaki, E., \& Anastassiadou, V. (2012). Elderly complete denture wearers: a social approach to tooth loss. Gerodontology, 29(2), e721-e727. Recuperado em 01 junho, 2015, de: doi: 10.1111/j.1741-2358.2011.00550.x.

Pistorius, J., Horn, J. G., Pistorius, A., \& Kraft, J. (2013). Oral health-related quality of life in patients with removable dentures. Schweiz Monatsschr fur Zahnmed=Revue mensuelle suisse d'odonto-stomatologie=Rivista mensile svizzera di odontologia $e$ stomatologia/SSO, 123(11), 964-971.

Polsani, L. R., Ajay Kumar, G., Githanjali, M., \& Anjana, R. (2011). Geriatric Psychology and Prosthodontic Patient. Int J Prost Restorative Dentistry, 1(1), 1-5. Recuperado em 01 junho, 2015, de: http://www.jaypeejournals.com/eJournals/ShowText.aspx?ID=1340\&Type=FREE\&TY $\mathrm{P}=\mathrm{TOP} \& \mathrm{IN}=$ eJournals/images/JPLOGO.gif \&IID=114\&isPDF=YES. 
Ruffino Netto, A. (1992). Qualidade de Vida: compromisso histórico da epidemiologia. Saúde em Debate, 35, 63-67. Recuperado em 01 junho, 2015, de: http://bases.bireme.br/cgibin/wxislind.exe/iah/online/?IsisScript=iah/iah.xis\&src=google\&base=LILACS\&lang $=p \&$ nextAction=lnk\&exprSearch=150848\&indexSearch=ID.

Soares, S. L. B., Rodrigues, R. A., Ribeiro, R. A., \& Rosendo, R. A. (2015). Avaliação dos usuários de prótese total, abordando aspectos funcionais, sociais e psicológicos. Revista Saúde \& Ciência Online, 4(2), 19-27. Recuperado em 01 dezembro, 2015, de: http://150.165.111.246/revistasaudeeciencia/index.php/RSC-UFCG/article/viewFile/250/164.

Sowmya, M. K., Vinaya, B., \& Krishna, P. (2011). Psychological impact of edentulousness. JIADS, 2(1), 34-36. Recuperado em 01 junho, 2015, de: https://scholar.google.com/citations?view_op=view_citation\&hl=en\&user=Svve1dkAA AAJ\&citation_for_view=Svve1dkAAAAJ:K1AtU1dfN6UC.

Yiengprugsawan, V., Somkotra, T., Seubsman, S. A., \& Sleigh, A. C. (2011). Oral Health-Related Quality of Life among a large national cohort of 87,134 Thai adults. Health and Quality of life Outcomes, 9(42), 1-8. Recuperado em 01 junho, 2015, de: doi: 10.1186/1477-7525-9-42.

Recebido em 12/11/2015

Aceito em 30/01/2016

Simone Kreve - Docente do Curso de Graduação em Odontologia da UNIPAR Universidade Paranaense Campus Cascavel. Cascavel, PR, Brasil.

E-mail: simonekreve@hotmail.com

Didier Anzolin - Docente do Curso de Graduação em Odontologia da UNIPAR Universidade Paranaense Campus Cascavel. Cascavel, PR, Brasil.

E-mail: didier@prof.unipar.br 